\title{
Editorial
}

\section{Advances in Nonlinear Vibration}

\author{
Livija Cveticanin, ${ }^{1}$ Kale Oyedeji, ${ }^{2}$ Vasile Marinca, ${ }^{3}$ and Nicolae Herisanu ${ }^{4}$ \\ ${ }^{1}$ Faculty of Technical Sciences, University of Novi Sad, Trg Dositeja Obradovica 6, 21000 Novi Sad, Serbia \\ ${ }^{2}$ Department of Physics, Morehouse College, Atlanta, GA, USA \\ ${ }^{3}$ Center for Advanced and Fundamental Technical Research, Romanian Academy, Timişoara Branch, Bulevardul Mihai Viteazu, \\ 24, 300223 Timişoara, Romania \\ ${ }^{4}$ Politehnica University of Timisoara, Bulevardul Mihai Viteazu, 1, 300222 Timişoara, Romania
}

Correspondence should be addressed to Livija Cveticanin; cveticanin@uns.ac.rs

Received 11 July 2013; Accepted 11 July 2013

Copyright ( $) 2013$ Livija Cveticanin et al. This is an open access article distributed under the Creative Commons Attribution License, which permits unrestricted use, distribution, and reproduction in any medium, provided the original work is properly cited.

\section{Introduction}

The main subject of the topic is nonlinear vibration and the oscillatory motion of the strong nonlinear system in all its varied aspects, including truly nonlinear oscillator, oscillator with time variable parameter, forced vibration, parametrical vibration, and analytical solving methods for these dynamic systems.

In this issue, 1 review and 8 original research papers are published. The papers analyze the oscillatory motion and vibration of the strong nonlinear oscillatory systems which appear and are applied in all the fields of science and engineering. The topics of the papers are

(i) strong nonlinear oscillatory systems (2 papers);

(ii) asymptotic methods in truly nonlinear oscillators (2 papers);

(iii) forced vibrations in the multiple-degree-of-freedom oscillatory system (1 paper);

(iv) application of the asymptotic methods in biomechanical (1 paper) solid structures (1 paper);

(v) nonlinear phenomena in stability and vibration of an elastic body (2 papers).

We are going to introduce the selected papers in the categories of nonlinear vibrations of a cubic-quintic Duffing oscillator, a biomechanical system, a truly nonlinear oscillator with its application to an electrical machine, multiple-degreeof-freedom oscillator and multibody system, and elastic body (sandwich beam and cylinder).

\section{Analytical Solution Procedures for the Cubic-Quintic Duffing Oscillator}

In the paper entitled "Analytical approximate solutions for the cubic-quintic Duffing oscillator in terms of elementary functions," A. Beléndez et al. present a procedure for solving the cubic-quintic Duffing oscillator. The method is based on the expansion of the restoring force into the Chebyshev polynomials and transformation of the original nonlinear differential equation into a cubic Duffing one. Approximate solutions are expressed in the form of the complete elliptic integral of the first kind and the cosine Jacobi elliptic function. Using the series expansion of the functions into elementary functions and applying the harmonic balance method, the periodic solution of the original nonlinear oscillator is obtained.

S. Durmaz and M. O. Kaya in their paper entitled "Highorder energy balance method to nonlinear oscillators" give another analytical procedure for solving the cubic-quintic Duffing oscillator. Namely, the energy balance method developed for high-order nonlinear oscillators is adopted for solving the cubic-quintic Duffing oscillator. The approximation is done up to the third order, and the maximal relative error of the frequency which decreases to $0.008 \%$ is analytically solved by applying the energy balance method. There is a 
good agreement of the analytically calculated frequencies and periodic solutions with the numerically calculated ones.

\section{Application of the Nonlinear Vibration Model to a Biomedical System}

In the paper "Review on mathematical and mechanical models of the vocal cord" by L. Cveticanin, a review on mathematical and mechanical models of the vocal cords is given. The basic model of the vocal cords, that is, vocal folds, is a two-mass nonlinear oscillator system which is assumed to be the basic one for mechanical description in voice production. The model is described with a system of two coupled secondorder differential equations. The two-mass model is modified into three, five, and more mass systems, systems with time variable parameters, and three-dimensional systems but also simplified into a one-mass system with coupled twodirection deflection function and with friction function. The corresponding mathematical models are the second-order nonlinear differential equations. The regular vibrations and the chaotic motion are investigated. Based on the obtained results, the pathology of vocal cords is determined.

\section{Vibration of a Truly Nonlinear Oscillator and Its Application in Electrical Machine}

In the paper of V. Marinca and N. Herişanu entitled "Optimal variational method for truly nonlinear oscillator," a novel analytical approximate solving technique is introduced. The main advantage of the method is that it allows the adjustment of convergence regions during calculation. Besides, the approach does not depend upon any small or large parameter. The suggested method is applied for solving the truly nonlinear oscillators. The analytically obtained solutions are compared with the numerical ones and show good agreement.

The paper entitled "An optimal approach to study the nonlinear behaviour of a rotating electrical machine" by N. Herişanu and V. Marinca considers the application of the optimal variational method for solving the oscillator with cubic nonlinearity and time variable coefficients. The procedure involves the presence of arbitrary convergencecontrol parameters which have to satisfy the condition of the optimal control. The method is applied for rotating electrical machines.

\section{Approximate Solution of a Multiple-Degree-of-Freedom Oscillator with Cubic Nonlinearity}

The paper entitled "Approximate super-and sub-harmonic response of a multi-DOFs system with local cubic nonlinearities under resonance" by Y. CaJin deals with the problem of multiple-degree-of-freedom dynamical system with cubic nonlinearities and with superharmonic or subharmonic excitation. The single modal natural resonance theory is applied for linearization and simplification in the system. While the system is controlled by multiple modes, modal analysis is used for linearization and to obtain the dominant modes. An example of a damped oscillator with ten degrees of freedom is considered. The approximate solutions are validated by comparing them with the results of direct numerical integration. The difference between solutions may be neglected.

In the paper of M. L. Scutaru and S. Vlase entitled "Some properties of motion equations describing the nonlinear dynamical response of a multibody system with flexible elements," the motion of a multibody system with flexible elements is considered. The system is discretized, and the motion is described with a system of strong nonlinear second-order differential equations with variable parameters. The linearization is done only for short time intervals. The coefficients are frozen and considered as constants. For such assumptions, the exact solution of the system is obtained, and based on the obtained result, the characteristics of the system are discussed.

\section{Vibration Properties of the Nonlinear Elastic Body}

In the "Nonlinear dynamics of an electrorheological sandwich beam with rotary oscillation," by K. Wei et al. and P. Xia, the nonlinear properties and the parametric instability of a rotating electrorheological sandwich beam with rotary oscillation are numerically analyzed. The system is described with a system of coupled nonlinear differential equations. The discretization is introduced, and the multiple scale method is applied for analyzing the parametric structural instability. The influence of the electrical field on the stability is investigated. It is concluded that the electrorheological materials have a significant influence on vibration characteristics and parametric instability. These materials can be used to adjust the stability of the rotating flexible beams.

Nonlinear periodical vibrations of cylinders are considered in "Nonlinear periodic oscillation of a cylindrical microvoid centered at an isotropic incompressible Ogden cylinder" by W. Zhang et al. The vibration of an infinitely long cylinder composed of an isotropic incompressible Ogden material with a microvoid at its center, where the outer surface of the cylinder is subjected to a uniform radial tensile load, is analyzed. Using the incompressibility condition and the boundary conditions, the motion of the microvoid is described with a second-order nonlinear ordinary differential equation. The qualitative analysis of the equation shows that two types of solutions exist: a nonlinear periodic oscillatory one and a blow-up solution. Namely, for certain parameter value of the incompressible Ogden material, there is a jump phenomenon in the amplitude-load diagram.

\section{Acknowledgment}

The guest editors would like to take this opportunity to thank all the contributions from the authors and reviewers.

\section{Livija Cveticanin Kale Oyedeji \\ Vasile Marinca \\ Nicolae Herisanu}




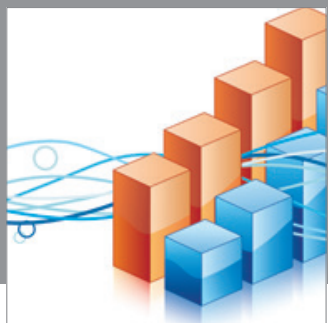

Advances in

Operations Research

mansans

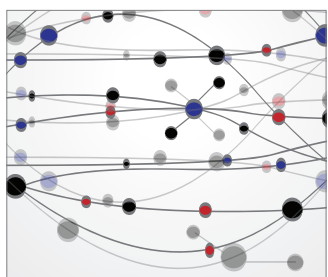

The Scientific World Journal
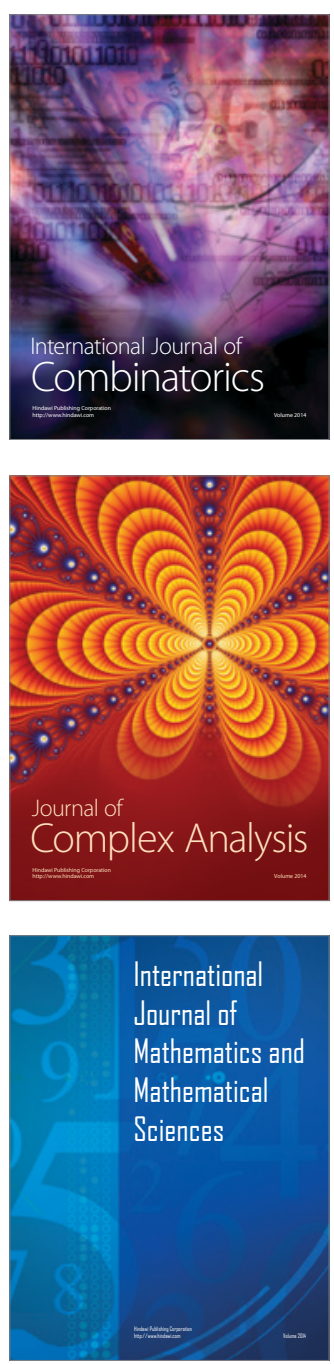
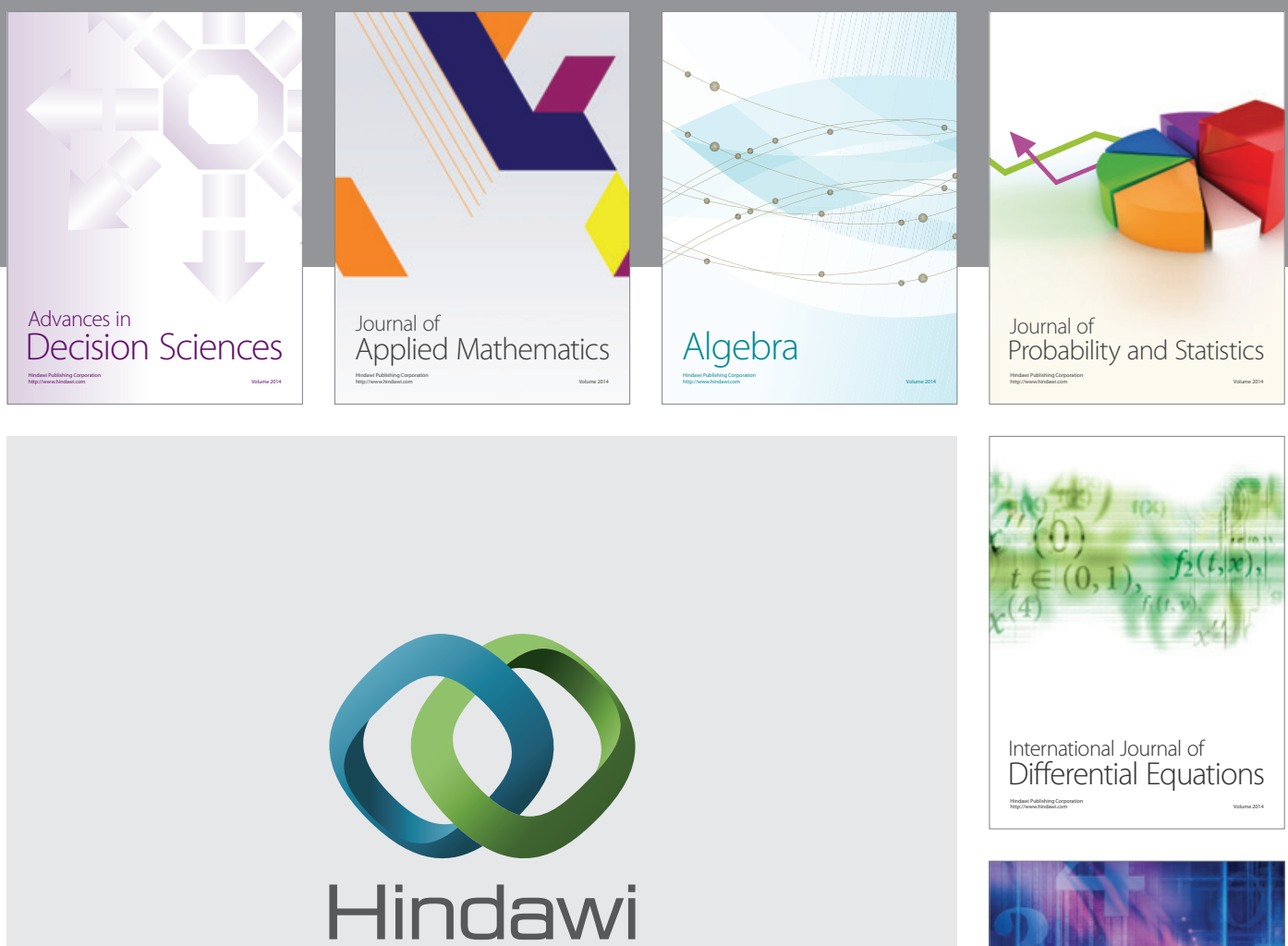

Submit your manuscripts at http://www.hindawi.com
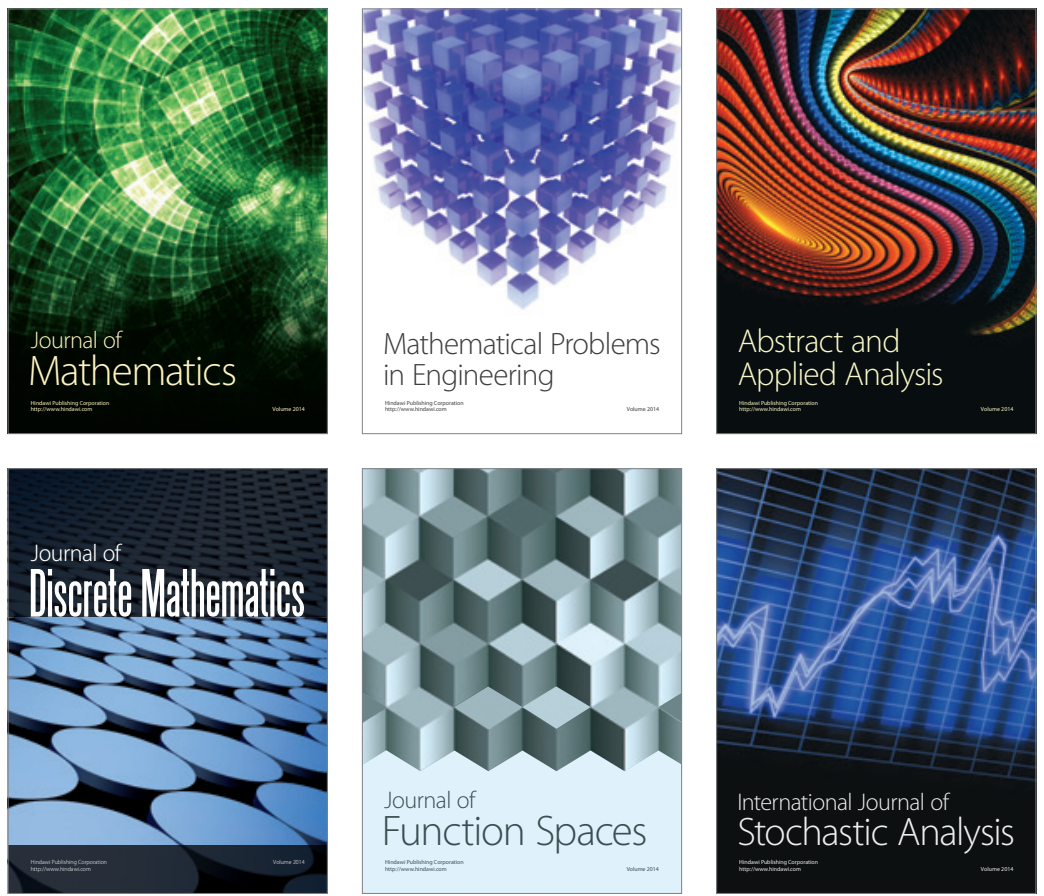

Journal of

Function Spaces

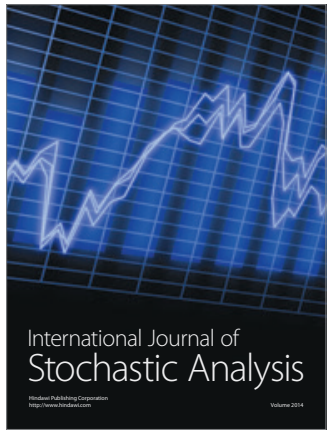

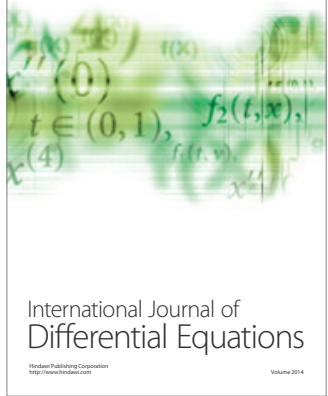
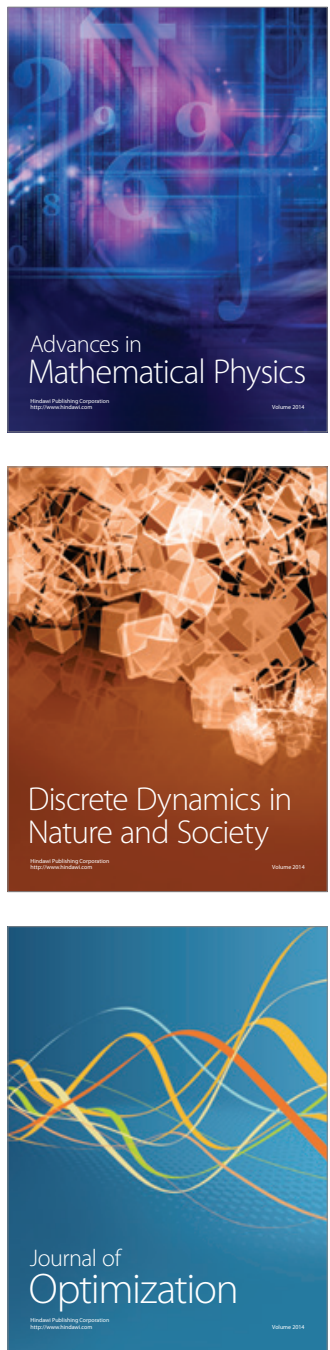